\title{
First-Line Immunotherapy in Advanced Non-Small-Cell Lung Cancer
}

Although immune checkpoint blockers (ICBs) were discovered in the 1990s, the initial reports of their efficacy in non-small-cell lung cancer (NSCLC) came through in 2012, when nivolumab (BMS-936558) showed responses in a Phase 1 study. From then on, there has been a constant flow of data pertaining ICBs in a multitude of previously treated malignancies.

ICBs have completely transformed care for untreated advanced NSCLC and now have entered the first-line therapeutic armamentarium based on convincing data. An encouraging update of Keynote-001 presented at the 2019 American Society of Clinical Oncology meeting showed that $23.2 \%$ overall and $29.6 \%$ of patients with a programmed death ligand (PD-L1) staining of $>50 \%$ treated with pembrolizumab (P) upfront were alive at 5 years. We will be looking at some of the landmark trials of immunotherapy in treatment-naive NSCLC in relation to common clinical situations.

\section{Untreated Non-small-Cell Lung Cancer with Programmed Death ligand Staining $\geq \mathbf{5 0 \%}$}

In the Phase 3 Keynote-24, 305 patients with untreated advanced NSCLC with PD-L1 tumor proportion score $\geq 50 \%$ (22C3, Dako), with no sensitizing epidermal growth factor receptor (EGFR) mutations or anaplastic lymphoma kinase (ALK) translocations, and with no untreated central nervous system (CNS) metastases were randomized (1:1) to $\mathrm{P} 200 \mathrm{mg}$ once every 3 weeks for up to 35 cycles or histology-based platinum doublet (chemotherapy [CT]) for 4-6 cycles.

The response rates favored $\mathrm{P}(44.8 \%$ vs. $27.8 \%)$. The median progression-free survival (PFS) was 4.3 months more with P (10.3 vs. 6 months, $P<0.001$, hazard ratio [HR]: 0.50), whereas the 12 -month PFS was $48 \%$ versus $15 \%$.

Similarly, the median overall survival (OS) was 30 months versus 14.2 months $(P<0.002$, HR: 0.63$)$, whereas the $24-$ month OS was $54 \%$ versus $34.5 \%$, an increment of about $20 \%$ at 2 years.

Based on these findings, as of now, the most prudent choice for this patient subgroup is monotherapy with P. Although there may be a consideration for chemo-immunotherapy in severely symptomatic high-risk patients, there is no head-to-head comparison between $\mathrm{P}$ alone and with $\mathrm{CT}$ in this population.

About $30 \%$ of advanced NSCLC patients who have this level of PD-L1 expression are eligible for this strategy.

Although cross-trial comparisons can be misleading, the outcomes of PD-L1 high subgroup of Keynote-189 with chemo-immunotherapy were comparable to those in $P$ arm of Keynote-24, with the 12-month OS rate being about $70 \%$ in both trials. Still, this will remain a point of contention awaiting concrete evidence.

The Keynote-042 was a similar study in patients with PD-L1 staining $\geq 1 \%$; although it did show a similar benefit of $\mathrm{P}$ monotherapy, the benefit was primarily driven by PD-L1-high patients. Thus, $\mathrm{P}$ monotherapy remains a less suitable choice for PD-L1 $<50 \%$ and combination approaches need to be utilized in such a scenario.

\section{Untreated Nonsquamous Non-Small-Cell Lung Cancer with Programmed Death Ligand Staining $<\mathbf{5 0 \%}$}

The Keynote-189 randomized 616 patients with untreated Stage IV nonsquamous NSCLC with no actionable EGFR/ALK alterations and no symptomatic CNS metastases to pemetrexed $(\mathrm{Pem})+$ platinum + placebo versus Pem + platinum agent $+\mathrm{P}$ followed by Pem $+\mathrm{P}$ maintenance (up to 35 cycles). The median PFS in the combination arm was 8.8 months versus 4.9 months in the $\mathrm{CT}$ arm $(P<0.001$, HR: 0.52). The 1-year OS was $73 \%$ versus $48.1 \%$, the benefit was apparent in all PD-L1 subgroups. An updated analysis showed a median OS of 22.0 versus 10.7 months $(P<0.00001$, HR: 0.56$)$. Despite $54 \%$ of CT-alone arm receiving ICB in second line, this difference was maintained. The PFS2 was also significantly longer (17 months vs. 9 months) in the combination arm.

Adverse events were $67.2 \%$ in combination group versus $65.8 \%$ in CT-placebo group.

Another noteworthy trial, IMpower150, studied addition of atezolizumab, a PD-L1 blocker, in combination with paclitaxel/carboplatin \pm bevacizumab (ACP vs. $\mathrm{ABCP}$ vs. $\mathrm{BCP}$ ) followed by maintenance. The median PFS of ABCP versus $\mathrm{BCP}$ was 8.3 months versus 6.8 months $(P<0.0001$, HR: 0.59 ), whereas the 18 -month PFS was $27 \%$ versus $8 \%$. The median OS was 19.8 versus 14.9 months (HR: 0.76). Importantly, patients with EGFR and ALK aberrations who had progressed or were intolerant to tyrosine-kinase inhibitor were not excluded from the study population. ABCP could represent a possible option for this subgroup.

Overall, in patients with PD-L1 expression $<50 \%$, a combination approach is more beneficial.

Untreated Squamous Non-Small-Cell Lung Cancer with Programmed Death Ligand Staining $<\mathbf{5 0 \%}$

The Keynote-407 randomized 559 patients with advanced squamous NSCLC to 
$\mathrm{P}+$ paclitaxel/nab-paclitaxel + carboplatin or paclitaxel/nab-paclitaxel + carboplatin + placebo $(\mathrm{P}+\mathrm{CT}$ followed by $\mathrm{P}$ for up to 31 cycles vs. CT). Again, the median OS favored the combination (15.9 vs. 11.3 months, $P<0.001$, HR: 0.64). The median PFS was similarly superior, 6.4 versus 4.8 months (HR: 0.56).

The Impower131 studied a similar population utilizing atezolizumab as the ICB. Although the median PFS was marginally better in the trial, an OS advantage could not be demonstrated.

Overall, combinations of ICBs and CT have been proven superior except in PD-L1-high patients. Although the Food and Drug Administration (FDA) has approved $\mathrm{P}$ as monotherapy in PD-L1 $>1 \%$, this may not be a wise choice in the PD-L1 $<50 \%$ cohort, while $\mathrm{P}$ monotherapy does remain the standard of care for PD-L1-high $(\geq 50 \%)$ population.

\section{I m m u n o- On cology-I m mu no - On cology Combination}

A recent update of Checkmate-227 studying nivolumab + ipilimumab versus platinum doublet in advanced NSCLC demonstrated an OS advantage for the immuno-oncology (IO) combination irrespective of PD-L1 expression, though the toxicity of IO-IO combination will be a concern with this approach.

In the end, and most importantly, we need to remember that these trials have included only up to Eastern Cooperative Oncology Group-performance status (PS) one patients with no actionable mutations. We really need to choose wisely and be cautious if we are going to extrapolate the available data to $\mathrm{PS}-2 / 3$ patients. In addition, $\mathrm{P}$ requires Dako PD-L1 immunohistochemistry 22C3 pharmDx assay as the PD-L1 testing platform as per the FDA approval. Toxicities of ICBs can be sometimes very serious and if not treated in time may be fatal, so careful preinfusion assessment, follow-up, monitoring, and patient and family education are always essential in the context of these drugs.

Financial support and sponsorship

Nil.

\section{Conflicts of interest}

There are no conflicts of interest.

\section{Vanita Noronha ${ }^{1}$, Prashant Mehta ${ }^{2}$, Prabhat Singh Malik ${ }^{3}$, Amol Patel ${ }^{4}$}

'Department of Medical Oncology, Tata Memorial Hospital, Mumbai, Maharashtra, ${ }^{2}$ Department of Medical Oncology/Hematology/BMT, Asian Institute of Medical Sciences, Faridabad, Haryana, ${ }^{3}$ Department of Medical Oncology, All India Institute of Medical Sciences, ${ }^{4}$ Malignant Diseases Treatment Centre, Army Hospital Research and Referral, New Delhi, India

Address for correspondence: Dr. Vanita Noronha, Department of Medical Oncology, Tata Memorial Hospital, Mumbai, Maharashtra, India.

\section{E-mail:vanita.noronha@gmail.com}

Submitted: 15 -Oct-2019

Revised: 19-Dec-2019

Accepted: 03-Jan-2020

Published: 17-Feb-2020

This is an open access journal, and articles are distributed under the terms of the Creative Commons Attribution-NonCommercial-ShareAlike 4.0 License, which allows others to remix, tweak, and build upon the work non-commercially, as long as appropriate credit is given and the new creations are licensed under the identical terms.

\begin{tabular}{|l|l|}
\hline \multicolumn{2}{|c|}{ Access this article online } \\
\hline Quick Response Code: & Website: \\
& www.ijmpo.org \\
\cline { 2 - 2 } & DOI: \\
\hline
\end{tabular}

How to cite this article: Noronha V, Mehta P, Malik PS, Patel A. First-line immunotherapy in advanced non-small-cell lung cancer. Indian $\mathrm{J}$ Med Paediatr Oncol 2019;40:461-2. 